\title{
Mathematical and Engineering Aspects of Chemically Reactive Tangent Hyperbolic Nanofluid over a Cone and Plate with Mixed Convection
}

\author{
Sardar Bilal, ${ }^{1}$ Zahir Shah $\mathbb{D}^{2},{ }^{2}$ Poom Kumam ${ }^{\mathbb{D}},{ }^{3,4,5}$ and Phatiphat Thounthong ${ }^{6}$ \\ ${ }^{1}$ Department of Mathematics, AIR University, Sector E-9 AIR Complex, P.O. 44000, Islamabad, Pakistan \\ ${ }^{2}$ Center of Excellence in Theoretical and Computational Science (TaCS-CoE), SCL 802 Fixed Point Laboratory, \\ Science Laboratory Building, King Mongkut's University of Technology Thonburi (KMUTT), 126, Bangkok, Thailand \\ ${ }^{3}$ KMUTT-Fixed Point Research Laboratory, Room SCL 802 Fixed Point Laboratory, Science Laboratory Building, \\ Department of Mathematics, Faculty of Science, King Mongkut's University of Technology Thonburi (KMUTT), \\ 126 Pracha-Uthit Road, Bang Mod, Thrung Khru, Bangkok 10140, Thailand \\ ${ }^{4}$ KMUTT-Fixed Point Theory and Applications Research Group, Theoretical and Computational Science Center (TaCS), \\ Science Laboratory Building, Faculty of Science, King Mongkut's University of Technology Thonburi (KMUTT), \\ 126 Pracha-Uthit Road, Bang Mod, Thrung Khru, Bangkok 10140, Thailand \\ ${ }^{5}$ Department of Medical Research, China Medical University Hospital, China Medical University, Taichung, Taiwan \\ ${ }^{6}$ Renewable Energy Research Centre, Department of Teacher Training in Electrical Engineering, Faculty of Technical Education, \\ King Mongkut's University of Technology North Bangkok, 1518 Pracharat 1 Road, Bangsue, Bangkok 10800, Thailand
}

Correspondence should be addressed to Poom Kumam; poom.kum@kmutt.ac.th

Received 28 October 2019; Revised 3 February 2020; Accepted 20 February 2020; Published 13 April 2020

Academic Editor: Vassilios C. Loukopoulos

Copyright (C) 2020 Sardar Bilal et al. This is an open access article distributed under the Creative Commons Attribution License, which permits unrestricted use, distribution, and reproduction in any medium, provided the original work is properly cited.

Current disquisition is expressed to elucidate the mathematical and engineering aspects of tangent hyperbolic liquid over a cone and plate which has valuable applications in industrial technological instruments such as cone and plate viscometer, spinning column technology, and biomass pyrolysis processing. The role of mixed convection and chemical reaction on physical features of fluid is considered. The attained coupled system is scrutinized by the aid of self-coded shooting method. In order to produce accuracy and refinement in the consequences, the coefficients of Runge-Kutta scheme are obliged. To get complete intellect about the current pagination, the impact of flow controlling variables on pertinent profiles are evaluated in quantitative and qualitative manner. The computations of wall drag force and heat and mass fluxes are presented pictorially and tabularly. From the thorough analysis, it is deduced that surface drag and convective thermal coefficient for cone is more in magnitude compared with those of plate. Similarly, dominance in thermal field is noted for cone compare with that in plate. Increment of velocity profile is revealed with enhancement in mixed convection parameter, whereas contrasting behavior is observed by varying Williamson and magnetic field parameters. Declining aptitude in thermal profile is exhibited against Prandtl number, whereas temperature of fluid mounts by changing the Brownian movement and thermophoresis parameters.

\section{Introduction}

Transmission of heat is the basic component of engineering which is concerned with the production, consumption, and transformation of energy. Heat transfer procedure has promising applications in thermal-exchanged systems in power houses, cooling and buildings, vehicles conditioner, and cooling system in processing plants. In all the aforementioned applications, heat is carried with the aid of diversified fluids and efficiency of each working system depends on strong influence of thermal conductivity. For such reasons, researchers are continuously working on the development of techniques to raise the thermal conductivity of utilized fluids. Initially, the idea of dispersing the particles 
into host fluid was proposed by Maxwell almost 120 years before. In this regard, Choi and Eastman [1] developed a new categorization of thermalized liquids depending on suspension of metallic nanoparticles with an average size of $100 \mathrm{~nm}$ termed as nanofluids. Masuda et al. [2] reconnoitered restricted thermal aptitudes of conventional fluids and added ultrafine elements in the base fluid for enhancement of thermal conductance. Irregular enhancement in thermal conductance of fluid with the addition of nanoconstituents in ethylene glycol-based fluid was bestowed by Eastman et al. [3]. Buongiorno [4] contemplated thermal attribute of nanofluids by examining seven distinctive properties possessed by nanofluids. Zhang et al. [5] explicated nanofluid flow generated by the variable stretched surface with radiation and MHD effects. Hsiao [6] scrutinized thermophysical features for viscoelastic fluid over a stretchable configuration. Sekholeslami et al. [7] accomplished an exploration on $\mathrm{CuO}$-water-based nanofluid by operating control volume-based finite element procedure. Malik et al. [8] scrutinized Casson nanofluid flow generated due to exponential cylinder and heeded the solution by implementing shooting procedure. Haq et al. [9] investigated thermophysical aspects of viscoelastic fluid generated due to the stretched surface. Aboalbashari et al. [10] executed thermophysical aspects in the attendance of slip velocity with Newtonian heating. They used four dissimilar types of nanoparticles to enhance thermophysical characteristics of base fluid. In the aforementioned literature, vast concern related to study of nanofluids with Newtonian and nonNewtonian has been addressed; however, for the sake of interest of readers, few communications are enclosed in [11-14].

Viscosity is the fundamental material property of each flowing object, and conceptualization about viscosity has an important role in the development of multiple fluids. On the basis of this intrinsic feature possessed by fluids, characterizations among them are made. Each unique material has its own behavior when subject to flow due to which they are classified into two main fields, i.e., viscous and in-viscous fluids. Viscous liquids are defined as the liquids in which deformation made by them is linear against appliance of the shear rate. The basic component of life, i.e., air and water are Newtonian fluids, so importance of such fluids cannot be neglected. On the contrary to this, in many cases, nonNewtonian model must be modelled. The joint analysis of tangent hyperbolic fluid over cone and plate has promising real world application such as in cone and plate viscometer which measures the viscosity of coatings, paints, and emulsions. In addition, another most attracting application of the current work is in spinning column technology which is related to the removal of volatile organic compounds from highly viscous polymeric suspensions. Such situation also arises in mining industries where mud and slurries are handled, chemical food industries where different eatable products and other homemade objects are produced, plastic industries where paints emulsion, suspension, and lubrications are prepared, and in biomedical flows. So, the essence of non-Newtonian fluids from rheological and practical sense cannot be neglected. Furthermore, the fluid dynamists have proposed several fluid models, e.g., Williamson fluid model, tangent hyperbolic model, and Carreau model on the basis of their pseudoplastic nature because they are unable to explicate with Navier Stokes equation. Among these various presented pseudoplastic models tangent hyperbolic fluid have been given less focus. So, present effort is dedicated to predict the intrinsic features of the tangent hyperbolic fluid model. Starting from the pioneering work carried out by Sher et al. [15], probed two-dimensional (2D) magnetized tangent hyperbolic liquids induced by a stretchable sheet. Friedman et al. [16] explained the practical implementation of tangent hyperbolic fluid by describing their utilization in damper coils. Nadeem and Akram [17] scrutinized hyperbolic liquid in asymmetric configuration. Naseer et al. [18] studied thermal aspects of tangent liquid generated by exponential axially stretched cylinder. Jyothi et al. [19] explicated peristaltic movement of tangent liquid through Darcy medium in symmetric channel.

Collaboration of boundary layer theory with electromagnetic forces has attracted promising attendance of researchers. Lorentz was the premier to present the mathematical expression for transversely applied magnetic field by proposing Lorentz force law to measure intensification of magnetic field in fluid flow. As an outcome, Maxwell electromagnetic equation is generated and coupling of it with Navier Stokes equation brings revolution in fluid dynamics. Alfeven [20] followed the concept manipulated by Lorentz and performed mathematical modeling about magnetohydrodynamic (MHD) flows. In recent years, tremendous work commenced on magnetohydrodynamic flow was conducted. Akbar et al. [21] measured reduction in velocity of tangent fluid produced by stretching of surface under the effect of perpendicularly executed MHD. Tain et al. [22] portrayed impact of Lorentz field on viscous liquid along with variable viscosity flow over a stretched surface. Malik et al. [23] evaluated influence of MHD on EyringPowel nanofluid caused by the stretched surface. Malik et al. [24] presented investigation about magnetization on viscoelastic fluid over a variably thickened stretching sheet. Bilal et al. [25] reconnoitered features of viscoelastic liquid over nonlinearly stretched surface under the effectiveness of magnetization. Analysis on behavior of hyperbolic liquid in the attendance of magnetic field and internal friction between molecules was manifested by Salahuddin et al. [26]. Some recent studies regarding MHD flow is referred in [27-31].

Inspired from previously acquired literature and reported applications about the pseudoplastic material, present pagination is different and innovative in such a way that it presents the analysis of such type of materials on two configurations jointly, i.e., cone and plate. So, current work excogitates mixed convection tangent hyperbolic flow along with the impact of chemical reaction on proposed joint configuration. According to author's knowledge, such type of work has not been executed so far. Firstly, the nonlinear differential equations concerning with flow fields are written in the form of nonlinear (ordinary differential equations) ODE's by employing permissible transmutations. The attained differential structure is handled by applying 
Runge-Kutta (RK) fifth-order method. Fascinating features of influential variables are disclosed tabularly and pictorially. Interpretation regarding the control of multiple engineering factors such as wall friction, heat transfer, and mass flux are also executed. In addition, the assurance of computed data is performed by constructing comparison with the published literature. So, authors have hope that this communication will intend research fraternity towards this direction.

\section{Model Development}

Consider two-dimensional (2D), time-independent, and magnetized incompressible hyperbolic tangent nanofluid caused by a cone/plate. Variable magnetic field $B(x)=B_{0} / x(G r)^{1 / 2}$ is employed alongside $x$-direction, as portrayed in Figure 1 (see Figures 2 and 3 ).

Constitutive conservation equations for tangent hyperbolic fluid are given as

$$
\begin{aligned}
& \operatorname{div} V=0, \\
& \rho \frac{\mathrm{d} V}{\mathrm{~d} t}=\operatorname{div} S+\rho b .
\end{aligned}
$$

The tensorial structure of fluid model is given as

$$
\begin{aligned}
& S=p I+\tau, \\
& \tau=-\left[\eta_{\infty}+\left(\eta_{0}-\eta_{\infty}\right) \tanh (\Gamma \dot{\gamma})^{n}\right] \dot{\gamma} .
\end{aligned}
$$

$\dot{\gamma}$ is defined as follows:

$$
\begin{aligned}
\dot{\gamma} & =\sqrt{\frac{1}{2} \Pi}, \\
\Pi & =\frac{1}{2} \operatorname{trac}\left[\operatorname{grad} V+(\operatorname{grad} V)^{t}\right]^{2} .
\end{aligned}
$$

By using $\eta_{\infty}=0$ and $\Gamma \dot{\gamma}<1$, the component of extrastress tensor is expressed as

$$
\begin{aligned}
\tau & =-\eta_{o}(\Gamma \dot{\gamma})^{n} \dot{\gamma}, \\
\tau & =-\eta_{o}(1+\Gamma \dot{\gamma}-1)^{n} \dot{\gamma}, \\
\tau & =-\eta_{o}(1+n[\Gamma \dot{\gamma}-1]) \dot{\gamma}, \\
\frac{\partial(r u)}{\partial x}+\frac{\partial(r v)}{\partial y}= & 0, \\
u \frac{\partial u}{\partial x}+v \frac{\partial u}{\partial r}= & \nu(1-n) \frac{\partial^{2} u}{\partial y^{2}}+\nu n \Gamma \sqrt{2} \frac{\partial u}{\partial y} \frac{\partial^{2} u}{\partial y^{2}} \\
\rho c_{p}\left(u \frac{\partial T}{\partial x}+v \frac{\partial T}{\partial y}\right)= & \alpha \frac{\partial^{2} T}{\partial y^{2}}+\tau\left(\frac{D_{T}}{T_{\infty}}\left(\frac{\partial T}{\partial y}\right)^{2}+D_{B} \frac{\partial T}{\partial y} \frac{\partial C}{\partial y}\right), \\
u \frac{\partial C}{\partial x}+v \frac{\partial C}{\partial y}= & D_{B} \frac{\partial^{2} C}{\partial y^{2}}+\frac{D_{T}}{T_{\infty}}\left(\frac{\partial^{2} T}{\partial y^{2}}\right)-k_{r}\left(C-C_{\infty}\right),
\end{aligned}
$$

subjected to the boundary conditions (BC's)

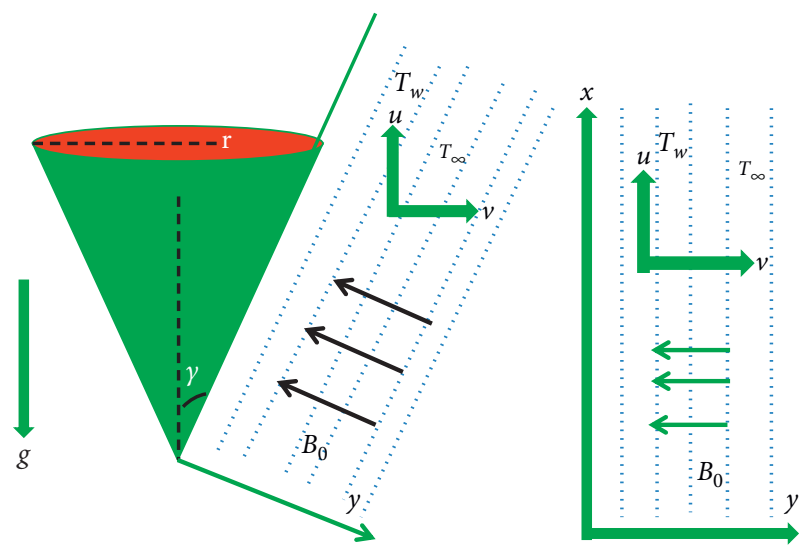

Figure 1: Physical Configuration.

$$
\begin{aligned}
& u=0, v=0, \quad \text { at } y=0, \text { as } u \longrightarrow 0, \\
& T=T_{w}, C=C_{w}, \quad T \longrightarrow T_{\infty}, C \longrightarrow C_{\infty}, \text { as } y \longrightarrow \infty .
\end{aligned}
$$

By using similarity transformations,

$$
\begin{aligned}
\eta & =\frac{y}{x}(G r)^{1 / 4}, \\
T & =T_{\infty}+\left(T_{w}-T_{\infty}\right) \theta(\eta), \\
G r & =\frac{\rho g \beta_{\tau}\left(T_{w}-T_{\infty}\right)}{v^{2}}, \\
\theta(\eta) & =\frac{T-T_{\infty}}{T_{w}-T_{\infty}}, \\
\phi(\eta) & =\frac{C-C_{\infty}}{C_{w}-C_{\infty}}, \\
u & =\frac{v}{x}(G r)^{1 / 2} f^{\prime}(\eta), \\
v & =-\frac{v}{x}(G r)^{1 / 4}\left(\frac{\eta}{4} f^{\prime}(\eta)-\frac{1}{4} f^{\prime}(\eta)\right),
\end{aligned}
$$

where $\eta$ is the using variable similarity.

The transformed expression are defined as follows and referred to [24] and [26]:

$$
\begin{aligned}
& (1-n) f^{\prime \prime \prime}+\frac{f f^{\prime \prime}}{4}-\frac{f^{\prime 2}}{2}+n W e f^{\prime \prime} f^{\prime \prime \prime}-(M+K) f^{\prime}+\lambda \theta=0 \\
& \theta^{\prime \prime}+\frac{\operatorname{Pr}}{4} \theta^{\prime} f+\operatorname{Pr} \theta^{\prime} \phi^{\prime} N_{b}+\operatorname{Pr} N_{t} \theta^{2}=0 \\
& \phi^{\prime \prime}+\frac{L e}{4} f \phi^{\prime}+\frac{N_{t}}{N_{b}} \theta^{\prime \prime}+\operatorname{Le\gamma } \phi=0
\end{aligned}
$$

The related transformed boundary conditions are 


$$
\begin{aligned}
& f(0)=0, f^{\prime}(0)=0, \theta(0)=1, \phi(0)=1, \quad \text { at } \eta=0, \\
& f^{\prime}(\infty)=0, \theta(\infty)=0, \phi(\infty)=0, \quad \text { at } \eta \longrightarrow \infty, \\
& \operatorname{Pr}=\frac{\nu}{\alpha} \\
& W e=\frac{v}{x^{2}} 2 \Gamma(G r)^{3 / 4} \\
& M=\frac{\sigma B_{0}^{2} x^{2}}{(G r)^{1 / 2} \rho}, \\
& K=\frac{x^{2}}{k(G r)^{1 / 2}} \\
& \lambda=\frac{g \beta_{T}\left(T_{w}-T_{\infty}\right) \cos \gamma}{v^{2}(G r)}, \\
& N_{b}=\frac{\tau D_{B}\left(C_{w}-C_{\infty}\right)}{v} \\
& N_{t}=\frac{\tau D_{t}\left(T_{w}-T_{\infty}\right)}{\nu T_{\infty}} \\
& L e=\frac{v}{D_{B}}, \\
& \gamma=\frac{k_{r}}{\rho g \beta_{\tau}}
\end{aligned}
$$

The wall shear factor and is given by

$$
\begin{aligned}
\tau_{w} & =\mu\left((1-n) \frac{\partial u}{\partial r}+\frac{n \Gamma}{\sqrt{2}}\left(\frac{\partial u}{\partial r}\right)^{2}\right), \\
q_{w} & =-k\left(\frac{\partial T}{\partial y}\right) \\
q_{m} & =-k\left(\frac{\partial C}{\partial y}\right) \\
\text { at } y & =0 \\
\frac{C_{f} \operatorname{Re}_{x}^{1 / 2}}{2} & =\left[(1-n) f^{\prime \prime}(0)+n W e f^{\prime 2}(0)\right]
\end{aligned}
$$

where $\operatorname{Re}_{x}^{1 / 2}=\left(\nu \mu / x^{2}\right)(G r)^{3 / 4}$.

Local Nusselt $(\mathrm{Nu})$ and Sherwood numbers $(\mathrm{Sh})$ are given by

$$
\begin{aligned}
N u & =-\theta^{\prime}(0), \\
S h & =-\phi^{\prime}(0),
\end{aligned}
$$

where Third Affiliated Hospital of Sun Yat-Sen University.

\section{Implemented Methodology}

From the overview of the literature, an extensive number of methodologies are proposed to handle fluid dynamic problems. Like in other current analysis is modelled in form of differential equations, which is easier to handle by way of shooting method in collaboration with the RK-Fehlberg scheme. The schematic diagram is shown in Figure 2. The schematic diagram is shown in Figure 3. Since it applies on initial value problem (IVP), so equations (7)-(9) are transmuted into $1^{\text {st }}$ order ODE's by letting $y_{1}=f, y_{2}=f^{\prime}, y_{3}=f^{\prime \prime}, \quad y_{4}=\theta, y_{5}=\theta^{\prime}, y_{6}=\phi, \quad$ and $y_{7}=\phi^{\prime}$; then, equations (7)-(9) are reduced to

$$
\begin{aligned}
& y_{1}^{\prime}=y_{2}, \\
& y_{2}^{\prime}=y_{3} \\
& y_{3}^{\prime}=\frac{1}{\left(1+W e^{2} y_{3}\right)}\left[-\left(\frac{y_{3} y_{1}}{4}-\frac{\left(y_{2}^{\prime}\right)^{2}}{2}\right)+(M+K) y_{2}-\lambda y_{4}\right] \\
& y_{4}^{\prime}=y_{5}, \\
& y_{5}^{\prime}=-\left[\frac{\operatorname{Pr}}{4} y_{1} y_{5}+\operatorname{Pr} y_{5} y_{7} N_{b}+\operatorname{Pr} N_{t} y_{5}^{2}\right] \\
& y_{6}^{\prime}=y_{7}, \\
& y_{7}^{\prime}=-\left[+\frac{L e}{4} y_{1} y_{7}+\frac{N_{t}}{N_{b}} y_{5}^{\prime}+\operatorname{Le\gamma } y_{6}\right] .
\end{aligned}
$$

The boundary conditions become

$$
\begin{array}{r}
y_{1}(0)=0, y_{2}(0)=0, y_{4}(0)=1, y_{6}(0)=1, \quad \text { at } \eta=0, \\
y_{2}(\infty) \longrightarrow 0, y_{4}(\infty) \longrightarrow 0, y_{6}(\infty) \longrightarrow 0, \quad \text { at } \eta \longrightarrow \infty .
\end{array}
$$

The involved central processing unit time is 16.7891 seconds. The computed solution will converge if boundary residuals $\left(R_{1}\left(u_{1}, u_{2}, u_{3}\right), R_{2}\left(u_{1}, u_{2}, u_{3}\right), R_{3}\left(u_{1}, u_{2}, u_{3}\right)\right)$ are less than tolerance error, i.e., $10^{-6}$. Otherwise, initial approximations are modified with Newton method and the process is repeated unless it meets the desired convergence criterion. Boundary residuals are defined such as

$$
\begin{aligned}
& R_{1}\left(u_{1}, u_{2}, u_{3}\right)=y_{2}(\infty)-\hat{y}_{2}(\infty), \\
& R_{2}\left(u_{1}, u_{2}, u_{3}\right)=y_{4}(\infty)-\widehat{y}_{4}(\infty), \\
& R_{3}\left(u_{1}, u_{2}, u_{3}\right)=y_{6}(\infty)-\hat{y}_{6}(\infty),
\end{aligned}
$$




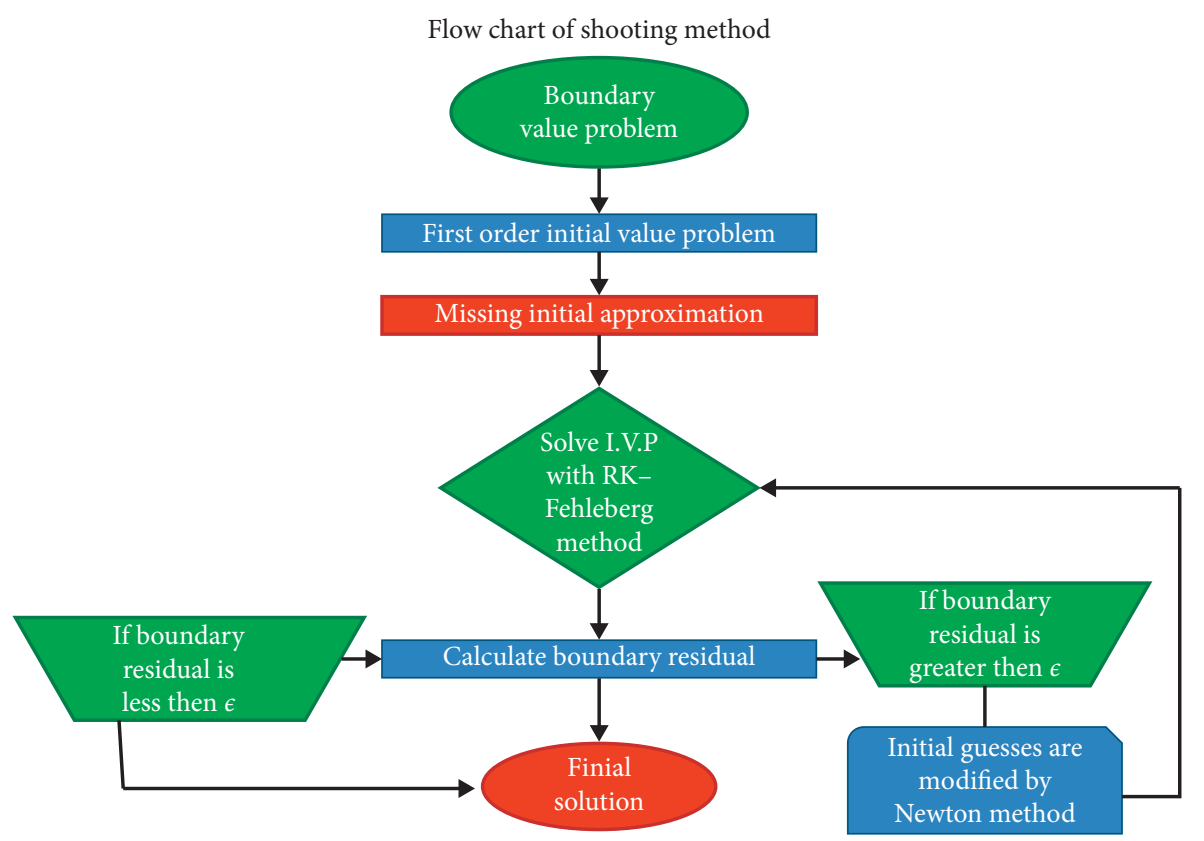

FIGURE 2: Schematic diagram of the shooting method.

where $\widehat{y}_{2}(\infty), \widehat{y}_{4}(\infty)$, and $\widehat{y}_{6}(\infty)$ are computed boundary values.

\section{Graphs}

4.1. Interpretation of Results. This segment is dedicated to explicate the impact of primitive parameters on concerning profiles such as momentum, thermal, mass, wall shear stress, heat flux, and mass flux distributions. Firstly, the equations are modelled by using rheological and conservation laws for the flow problem. Similarity transformations are obliged to convert the attained system of differential equations into ordinary differential form to execute solution. RK-Fehlberg method in collaboration with shooting scheme is implemented to manipulate result from system of equations. Table 1 examines compatibility of present findings by establishing comparison with Anil and Roy [25]. Total exactness about consequences of local Nusselt number $-\theta^{\prime}(0)$ with the existing literature is manifested. From the physical point of view, the credibility of current work is also assured by the finding increasing aptitude in Nusselt number against uplifting magnitude of Pr. Tables 2-4 divulge numerical data about surface drag coefficient, heat flux, and mass flux coefficients against different parameters. Table 1 measures the variation in wall shear stress against magnetic parameter $M$ and porosity parameter $K$. It is found that, by increasing $M$ and $K$, the magnitude of skin friction coefficient for plate is more than in case of cone. It is justified physically that, for plate, the connection of fluid particles with the surface is more in comparison to cone. So, more resistance is offered to fluid particles in case of plate than cone. Table 2 expresses the extensive enhancement in convective heat transfer coefficient for plate in comparison to cone. Physically, it is because of the reason that more contact is made by fluid particles with plate surface and hence more heat is transferred. Table 3 also addresses similar increasing manner in mass flux coefficient for plate than in cone against different flow controlling parameters. Figure 3 shows the gometry of the problem. Figure 4 explains delineation in velocity with respect to magnetic parameter $M$ for fluid flow over a cone/plate. Physically, magnetic field parameter $M$ induces resistive forces in the flow and is always applied in a perpendicular direction to decrease the movement of fluid particles at high velocity. Figure 5 represents deceasing impact of Weissenberg number $W e$ on momentum profile for cone/plate cases. Physical justification behind this effect is that Weissenberg number $W e$ is the ratio of relaxation time to retardation time thus by increasing $W e$ the fluid particles takes more time to retain their original position and thus the velocity down surges. Figure 6 represents enhancing attribute in velocity against mixed convection parameter $\lambda$. Physically, due to increased mixed convection parameter, buoyancy forces are generated in the flow which creates temperature difference in flow domain. Thus, as an outcome, the velocity of fluid enriches. Figure 7 reveals diminishing impact on thermal distribution for intensification in magnitude to Pr. This is because of the fact that $\operatorname{Pr}$ expresses ratio of viscous to thermal diffusions and with the uplift of Pr thermal diffusion decrements and the temperature decreases. Figure 8 depicts the impact of $N_{t}$ on thermal profile. It is found that increases in thermophoresis parameter $N_{t}$ fluid moves from hot to cold region hence thermal profile of the fluid decreases. Figure 9 demonstrates the influence of Brownian movement parameter $N_{b}$ on thermal profile. It is evidenced that by enhancing the Brownian motion parameter $N_{b}$ the temperature profile enhances and boundary layer thickness reduced. Figure 10 depicts 


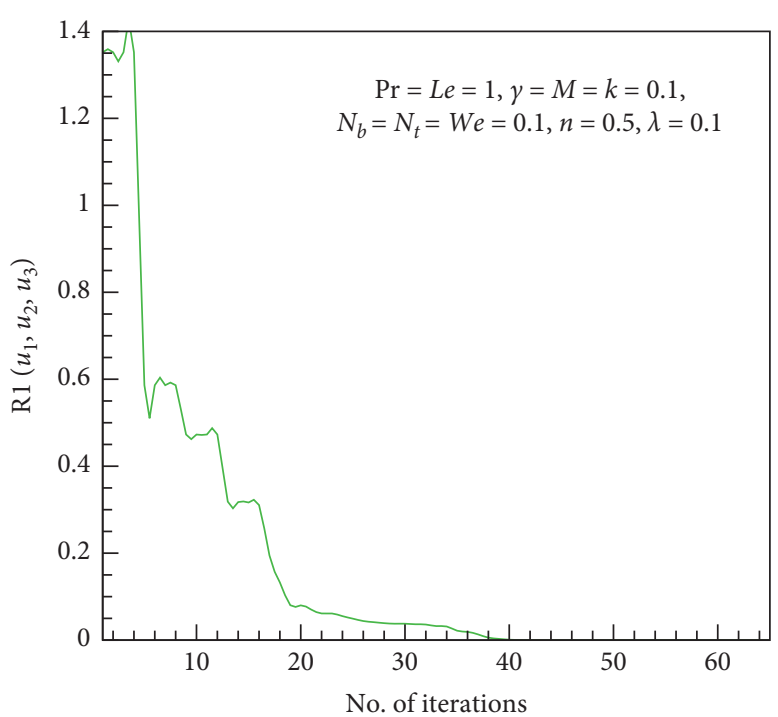

(a)

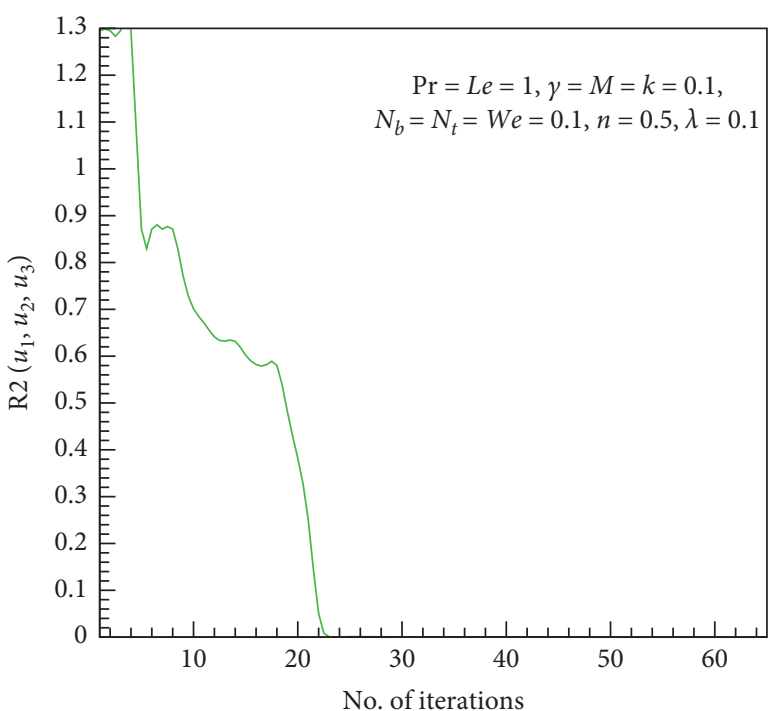

(b)

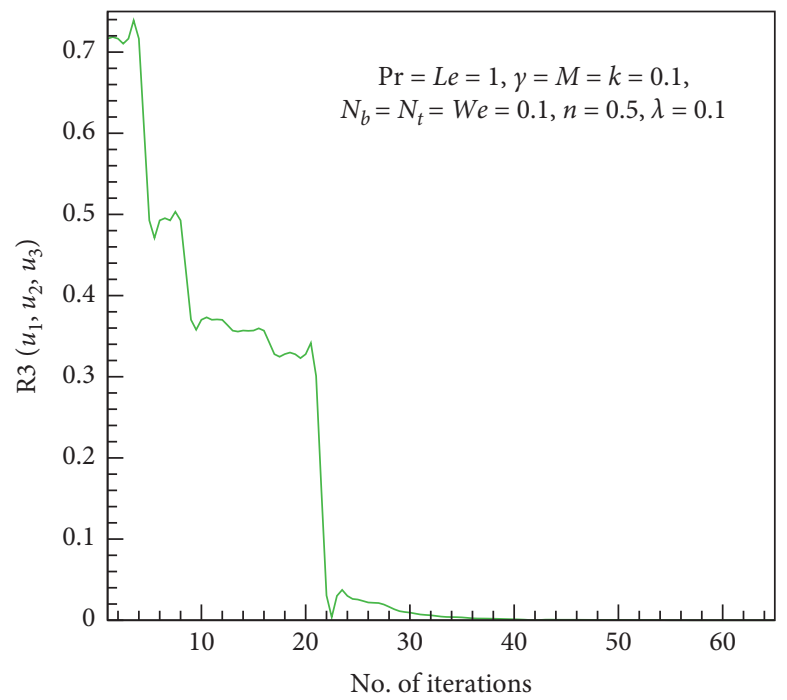

(c)

FIgURE 3: Computation of residual error for convergence.

TABLE 1: Comparison of the numerical results $-\theta^{\prime}(0)$ for magnitude of $\lambda$ and $\operatorname{Pr}$ when $\gamma=0, N_{t}=0, N_{b} \longrightarrow 0, L e=0, W e=0$, and $M=0$.

\begin{tabular}{cccc}
\hline $\operatorname{Pr}$ & $\lambda$ & Anil and Roy [25] & Present findings $\left[-\theta^{\prime}(0)\right]$ \\
\hline \multirow{3}{*}{0.7} & 0 & 0.4305 & 0.4586 \\
& 1 & 0.6127 & 0.6324 \\
& 10 & 1.0175 & 0.99843 \\
1 & 0 & 0.557294 & 0.55829 \\
& 1 & 0.721982 & 0.72318 \\
& 10 & 1.170983 & 1.17197 \\
10 & 0 & 1.4042 & 1.4342 \\
& 1 & 1.5885 & 1.5795 \\
& 10 & 2.3528 & 2.3988 \\
\hline
\end{tabular}

decreasing change in concentration distribution and associated boundary thickness against $N_{b}$. It is due to the fact that, by increasing Brownian motion parameter, the
TABle 2: Computation showing different values of skin friction coefficient $M$ and $k$.

\begin{tabular}{|c|c|c|c|}
\hline \multirow[b]{2}{*}{$M$} & \multirow[b]{2}{*}{$K$} & \multicolumn{2}{|c|}{$\frac{C_{f} \operatorname{Re}_{x}^{1 / 2}}{2}$} \\
\hline & & Cone & Plate \\
\hline & 0.1 & 0.1001 & 0.9708 \\
\hline \multirow[t]{2}{*}{0.1} & 0.2 & 0.0956 & 0.0957 \\
\hline & 0.3 & 0.0917 & 0.0918 \\
\hline \multirow[t]{3}{*}{0.5} & 0.1 & 0.0850 & 0.0852 \\
\hline & 0.2 & 0.0822 & 0.0824 \\
\hline & 0.3 & 0.0796 & 0.0798 \\
\hline \multirow[t]{3}{*}{0.9} & 0.1 & 0.0751 & 0.0753 \\
\hline & 0.2 & 0.0731 & 0.0733 \\
\hline & 0.3 & 0.0713 & 0.0715 \\
\hline
\end{tabular}

velocity of fluid particles increases and concentration decreases. Figure 11 discloses the reducing behavior of concentration profile against thermophoresis parameter $N_{t}$. By 
TABle 3: Numerical data of $-\theta^{\prime}(0)$ with variations of $\operatorname{Pr}$ and $N_{b}$ for cone and plate.

\begin{tabular}{cccc}
\hline & & \multicolumn{2}{c}{ Nusselt number } \\
$\operatorname{Pr}$ & $N_{b}$ & Cone & Plate \\
\hline \multirow{3}{*}{1.2} & 0.1 & 0.9044 & 0.5323 \\
& 0.2 & 0.7588 & 0.5074 \\
& 0.3 & 0.2618 & 0.4921 \\
1.4 & 0.1 & 0.2825 & 0.5442 \\
& 0.2 & 0.6546 & 0.5259 \\
& 0.3 & 0.2492 & 0.5079 \\
& 0.1 & 0.2747 & 0.5618 \\
& 0.2 & 0.3538 & 0.5408 \\
& 0.3 & 0.4713 & 0.5715 \\
\hline
\end{tabular}

TABle 4: Computation showing $-\phi^{\prime}(0) N_{b}=N_{t}=W e=M=\lambda=$ 0.1 and $\operatorname{Pr}=1$ with cone and plate.

\begin{tabular}{lccc}
\hline & & & $\varphi^{\prime}(0)$ \\
$\gamma$ & Le & Cone & Plate \\
\hline \multirow{3}{*}{0.1} & 0.1 & 0.7993 & 1.0046 \\
& 0.2 & 0.7873 & 0.9533 \\
0.3 & 0.3 & 0.7551 & 0.8977 \\
& 0.1 & 0.7865 & 0.9923 \\
& 0.2 & 0.7475 & 0.9109 \\
0.5 & 0.3 & 0.7062 & 0.8154 \\
& 0.1 & 0.7735 & 0.9798 \\
& 0.2 & 0.7053 & 0.8652 \\
& 0.3 & 0.6296 & 0.7183 \\
\hline
\end{tabular}

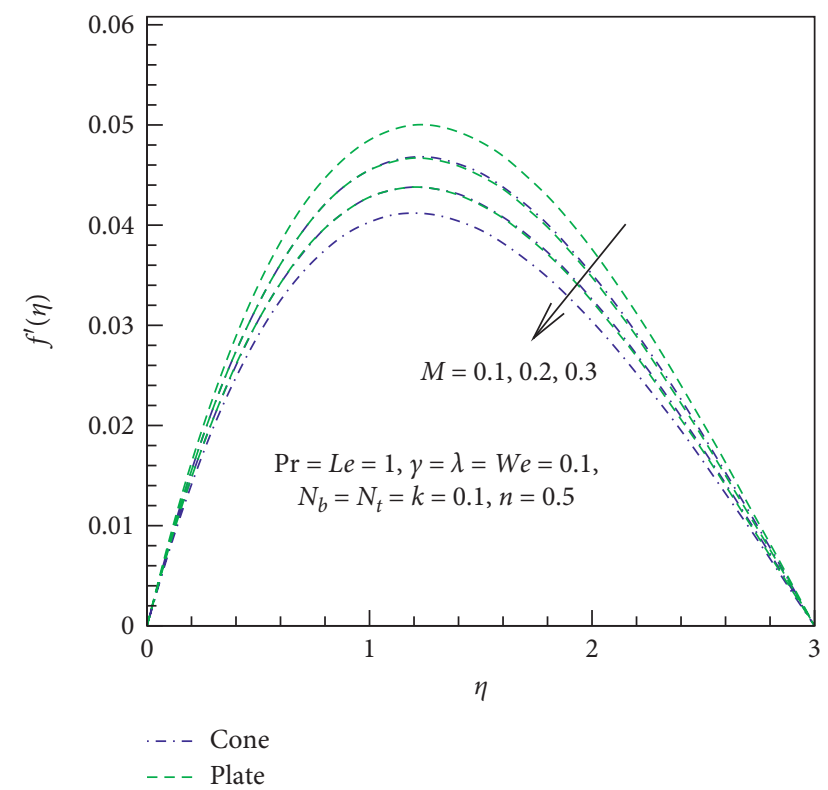

Figure 4: Bearing of $M$ on $f^{\prime}(\eta)$.

increasing thermophoresis parameter, the fluid particles move from hot to cold region and as an outcome the concentration profile decreases. Figure 12 describes the features of $\mathrm{Le}$ on mass distribution. It is clear that mass transfer increases with Lewis number $L e$ due to improvement in nanoparticles volume

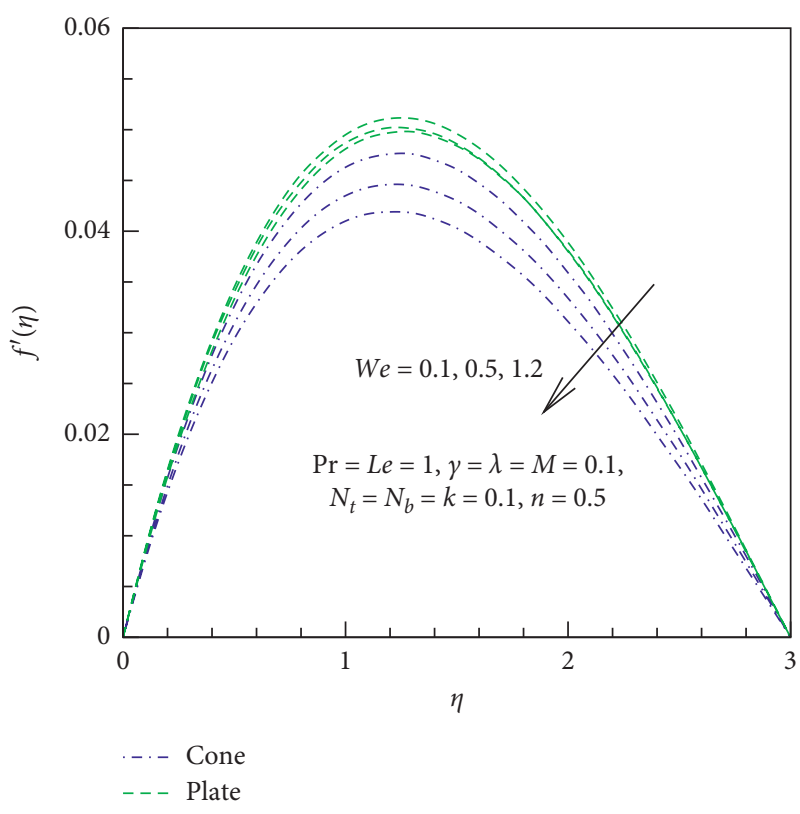

Figure 5: Bearing of We on $f^{\prime}(\eta)$.

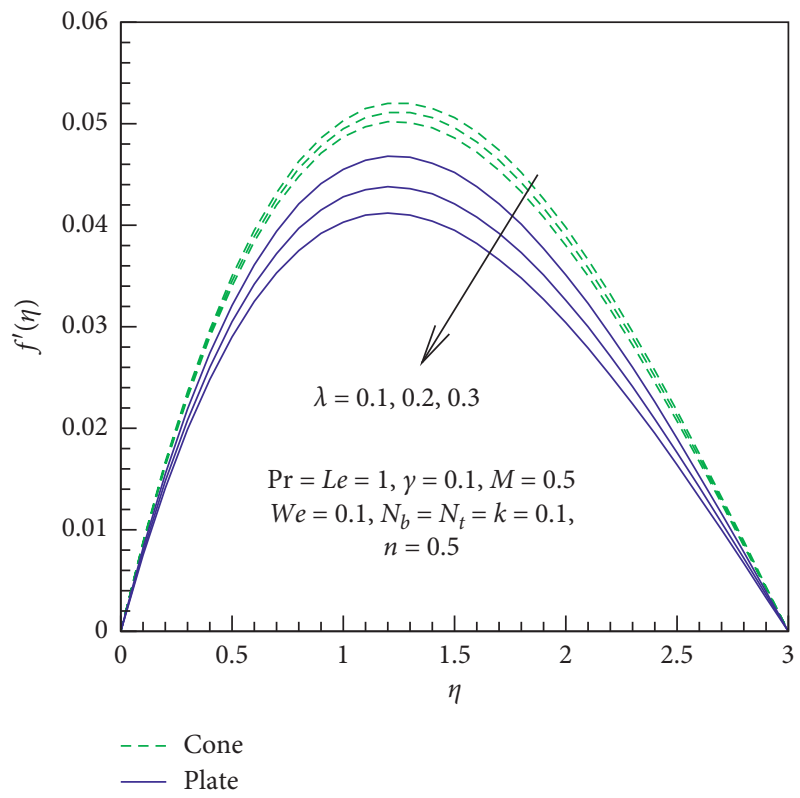

Figure 6: Bearing of $\lambda$ on $f^{\prime}(\eta)$.

fraction. Figure 13 explicates the impression of chemical reaction parameter $\gamma$ on the mass transfer rate. It is clear that, for large values of chemical reactions parameter $\gamma, \varphi(\eta)$ depreciates. The concentration profile reduces with increment in chemical reactions for $\gamma>0$ while chemical reaction has opposite behavior for $\gamma<0$. Figure 14 shows the role of magnetization $M$ and Weissenberg number We on wall drag coefficients. It is depicted that the skin friction coefficient grid as both parameters increases. Figure 15 depicts the effects of wall drag coefficients for various values of parameters $M$ and $\lambda$. This phenomenon causes the increase of skin friction with increase in $M$ 


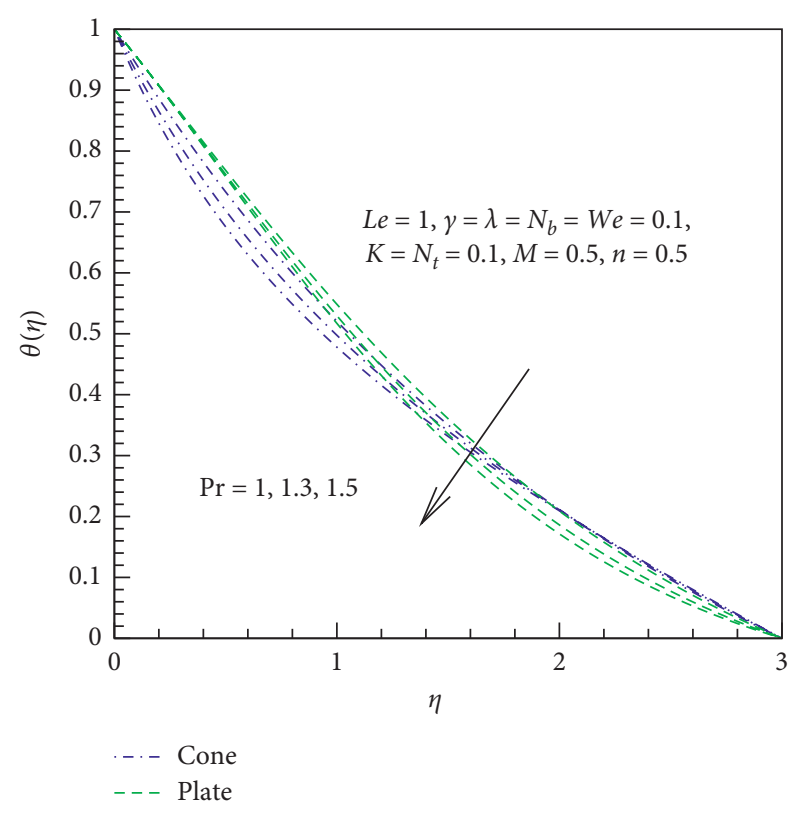

Figure 7: Bearing of Pr on $\theta(\eta)$.

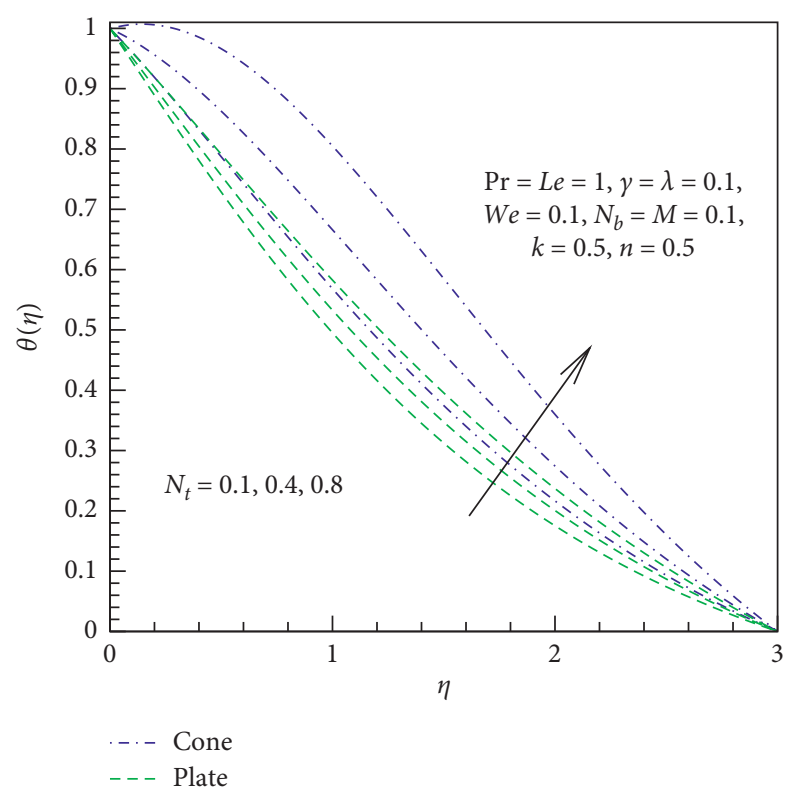

Figure 8: Bearing of $N_{t}$ on $\theta(\eta)$.

and $\lambda$. Figure 16 illustrated the variation of thermophoresis and Brownian moment parameter on the thermal transfer rate. It is observed that when $N_{t}$ is increased, the local thermal transfer rate reduced.

\section{Concluding Remarks}

This work is presented to disclose the features of mixed convection flow of tangent hyperbolic nanofluid over a cone/ plate in the presence of variable magnetic field strength, porosity aspects, and chemical reactions. Mathematical modeling of concerning problem is manifested in the form of partial differential expressions. The attained partial

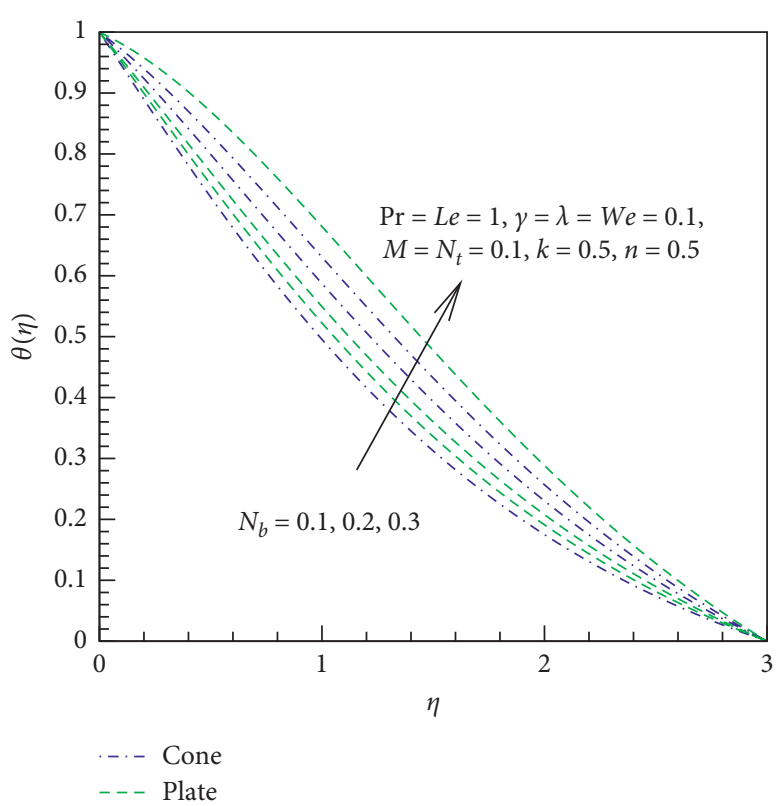

Figure 9: Bearing of $N_{b}$ on $\theta(\eta)$.

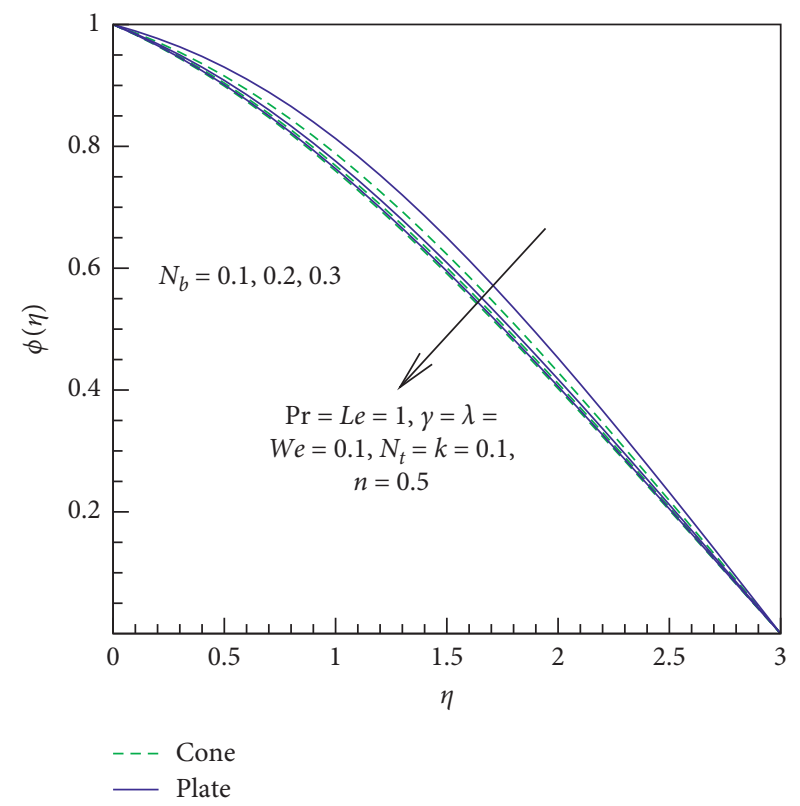

Figure 10: Bearing of $N_{b}$ on $\phi(\eta)$.

differential expressions are transmuted into ordinary differential equation by implementing similarity approach. Afterwards, the solution of attained equations is solved by implementing the shooting method along with the Runge-Kutta-Fehlberg method.

The following key findings have been derived from the analysis:

Magnitude of drag coefficient for plate is more than in the case of cone

Momentum distribution and associated boundary layer increase in case of cone than plate 


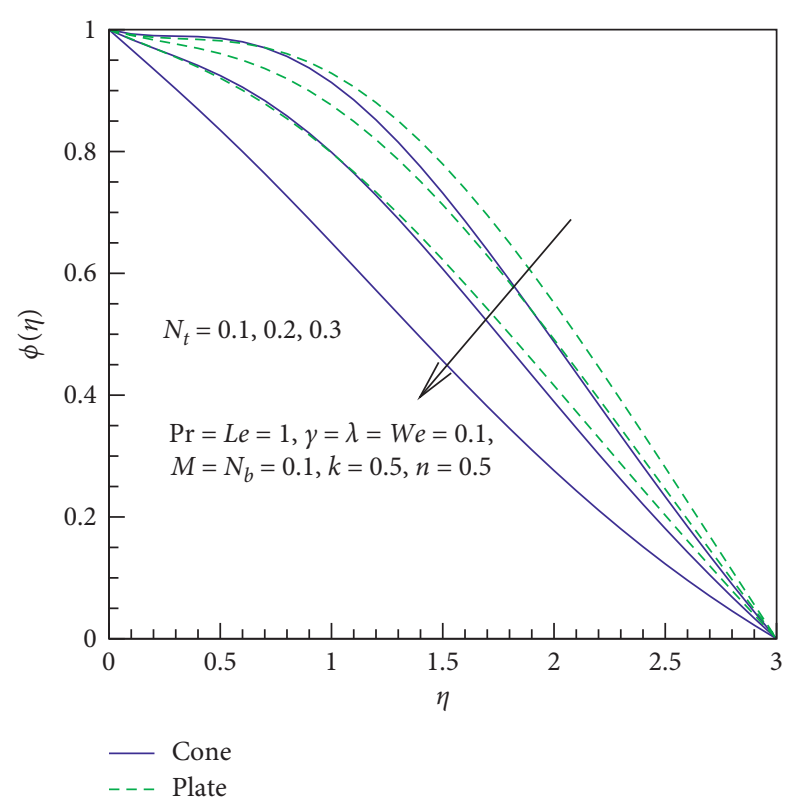

Figure 11: Bearing of $N_{t}$ on $\phi(\eta)$.

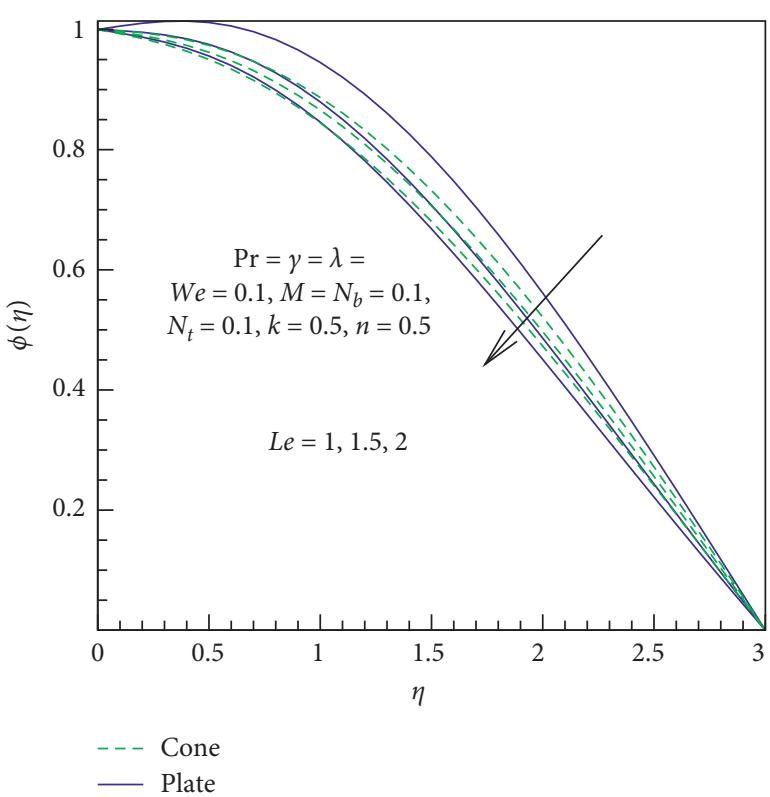

Figure 12: Bearing of Le on $\varphi(\eta)$.

Intensification in trends of thermal profile is manifested in cone compared with that of flat plate Incrementing trend in temperature against inciting values of $N_{b}$ and $N_{t}$ is depicted

Concentration profile shows decrement pattern against upsurging values of Brownian movement parameter $N_{b}$ and Lewis number Le

\section{Nomenclature}

$B(x): \quad$ Magnetic field along $x$-direction

$M: \quad$ Magnetic parameter

$B_{0}: \quad$ Strength of magnetic field

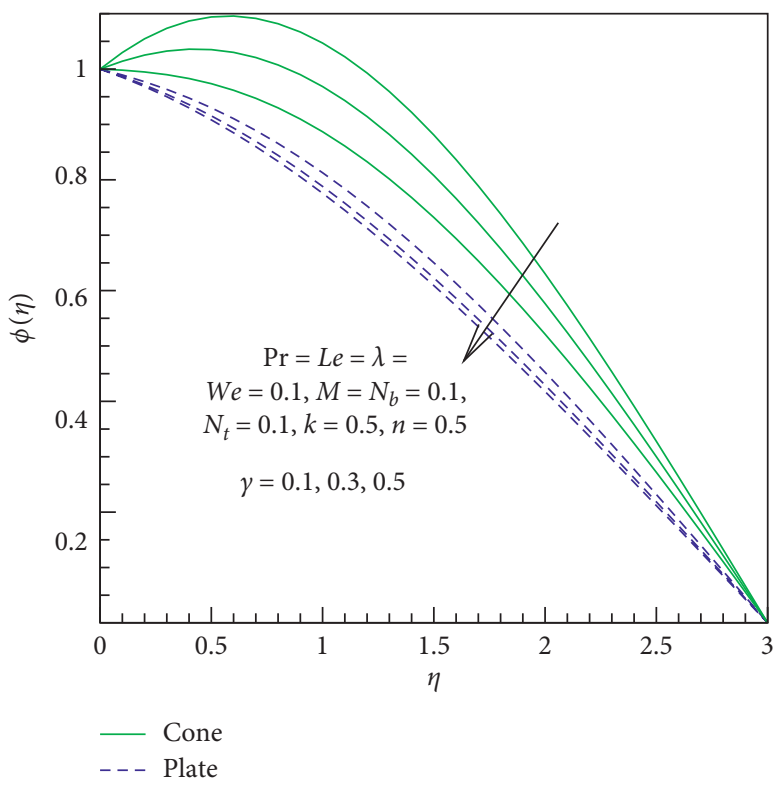

Figure 13: Bearing of $\gamma$ on $\varphi(\eta)$.

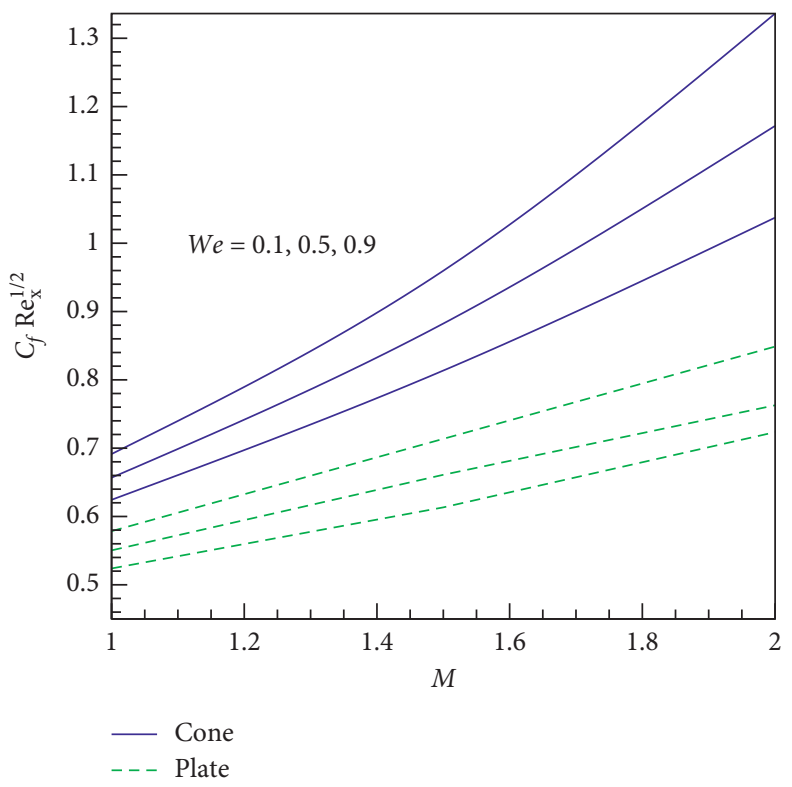

FIgURe 14: Influence $W e$ of skin friction.

Pr: $\quad$ Prandtl number

Gr: $\quad$ Grashof number

BCs: $\quad$ Boundary conditions

$\rho: \quad$ Density of fluid

RK: $\quad$ Runge-Kutta

$V: \quad$ Velocity vector

MHD: $\quad$ Magnetohydrodynamic

S: $\quad$ Cauchy stress tensor

2D: $\quad$ Two dimensional

$b$ : $\quad$ Body force

$n: \quad$ Power law index

$\mathrm{d} / \mathrm{d} t: \quad$ Material time derivative

We: Weissenberg number

$\tau$ : $\quad$ Extrastress tensor 


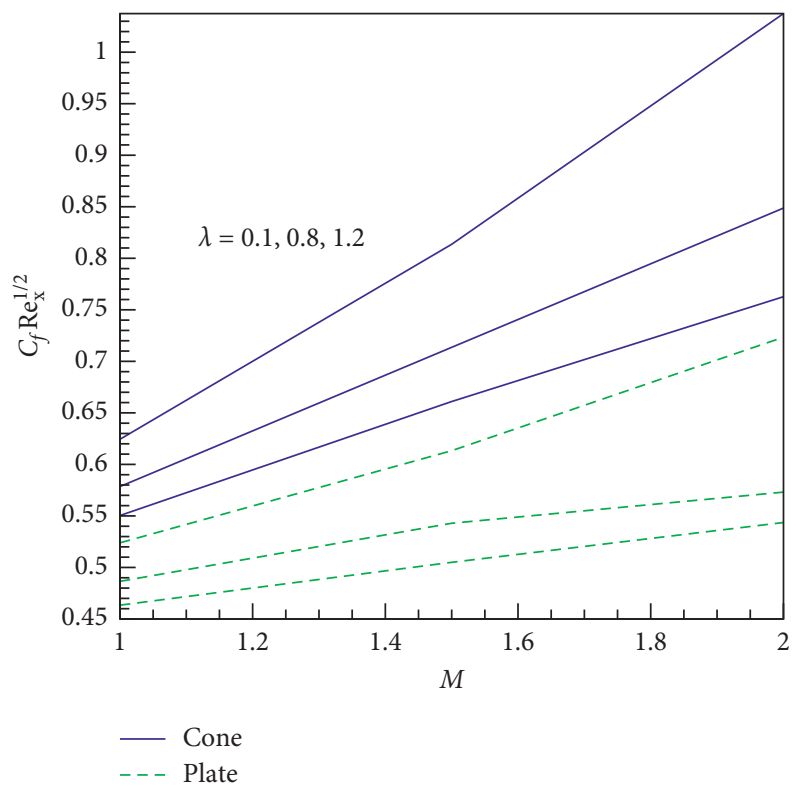

FIGURE 15: Influence of $\lambda$ on skin friction.

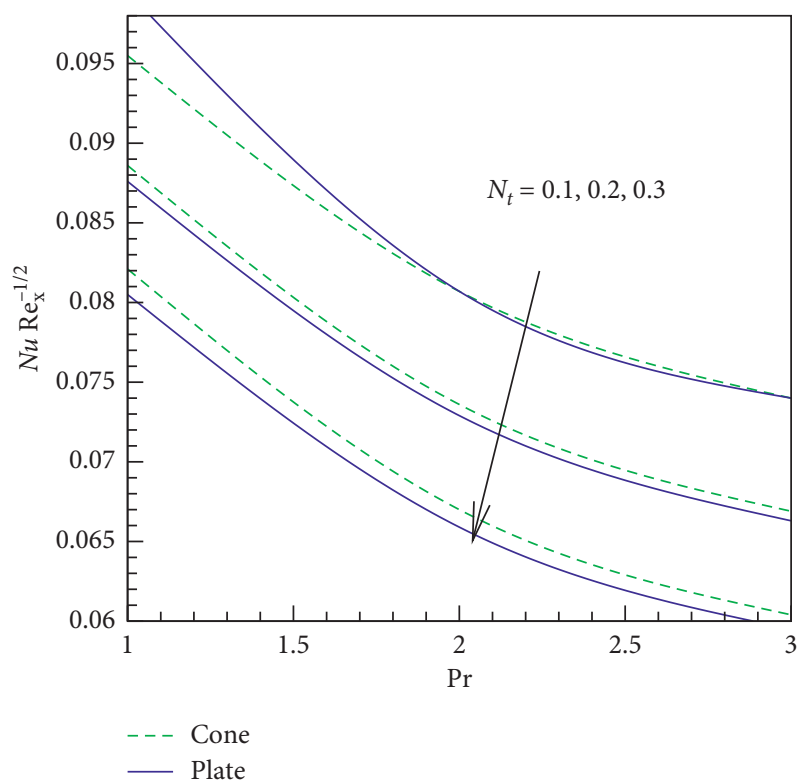

Figure 16: Bearing of $N_{t}$ on $-\theta^{\prime}(0)$.

$\lambda: \quad$ Mixed convection parameter

$\eta_{0}$ : $\quad$ Viscosity at zero shear stress

$N_{t}$ : Thermophoresis parameter

$\eta_{\infty}$ : $\quad$ Viscosity at infinite shear stress

$N_{b}$ : $\quad$ Brownian motion parameter

$\dot{\gamma}: \quad$ Time rate constant

Le: Lewis number

$A_{1}$ : $\quad$ First rivlin-ericksoe tensor

$\gamma: \quad$ Chemical reaction parameter

$\theta$ : Dimensionless temperature

$C_{f} \operatorname{Re}_{x}^{1 / 2}: \quad$ Skin friction coefficient

$\phi$ : Dimensionless concentration

$N u \operatorname{Re}_{x}^{-(1 / 2)}:$ Nusselt number

$\begin{array}{ll}T_{w}: & \text { Wall temperature } \\ \operatorname{ShRe}_{x}^{-(1 / 2)}: & \text { Sherwood number } \\ T_{\infty}: & \text { Ambient temperature } \\ \tau_{w}: & \text { Wall shear stress } \\ C_{w}: & \text { Wall concentration } \\ q_{w}: & \text { Wall heat flux. }\end{array}$

\section{Data Availability}

Data sharing not applicable to this article as no datasets were generated or analyzed during the current study.

\section{Conflicts of Interest}

The authors declare that they have no conflicts of interest.

\section{Authors' Contributions}

All authors equally contributed to this manuscript and approved the final version.

\section{Acknowledgments}

This project was supported by the Theoretical and Computational Science (TaCS) Center under Computational and Applied Science for Smart Innovation Research Cluster (CLASSIC), Faculty of Science, KMUTT.

\section{References}

[1] U. S. Choi and J. A. Eastman, "Enhancing thermal conductivity of fluids with nanoparticles," in Proceedings of the International Mechanical Engineering Congress and Exhibition, The American Society of Mechanical Engineers, San Francisco, CA, USA, November 1995.

[2] H. Masuda, A. Ebata, K. Teramae, and N. Hishinuma, "Alteration of thermal conductivity and viscosity of liquid by dispersing ultra-fine particles. Dispersion of $\mathrm{Al}_{2} \mathrm{O}_{3}, \mathrm{SiO}_{2}$ and $\mathrm{TiO}_{2}$ ultra-fine particles," Netsu Bussei, vol. 7, no. 4, pp. 227-233, 1993.

[3] J. A. Eastman, U. S. Choi, S. Li, L. J. Thompsonand, and S. Lee, "Enhanced thermal conductivity through the development of nanofluids," MRS Proceedings, vol. 457, no. 3, 1996.

[4] J. Buongiorno, "Convective transport in nanofluids," Journal of Heat Transfer, vol. 128, no. 3, pp. 240-250, 2006.

[5] C. Zhang, L. Zheng, X. Zhang, and G. Chen, "MHD flow and radiation heat transfer of nanofluids in porous media with variable surface heat flux and chemical reaction," Applied Mathematical Modelling, vol. 39, no. 1, pp. 164-181, 2015.

[6] K. L. Hsiao, "Stagnation electrical MHD nano-fluid mixed convection with slip boundary on a stretching sheet," Applied Thermal Engineering, vol. 98, pp. 850-861, 2016.

[7] M. Sheikholeslami, M. G. Bandpy, R. Ellahi, and A. Zeeshan, "Simulation of $\mathrm{MHD} \mathrm{CuO}$-water nano-fluid flow and convective heat transfer considering Lorentz forces," Journal of Magnetism and Magnetic Materials, vol. 369, pp. 69-80, 2014.

[8] M. Y. Malik, I. Khan, A. Hussain, and T. Salahuddin, "Mixed convection flow of MHD-eyring powell nano-fluid over a stretching sheet: a numerical study," AIP Advances, vol. 5, no. 11, Article ID 117118, 2015. 
[9] R. Haq, S. Nadeem, Z. H. Khan, and T. G. Okedyao, "Convective heat transfer and MHD effects on casson nano-fluid over a shrinking sheet," Central European Journal of Physics, vol. 12, pp. 862-871, 2014.

[10] M. H. Aboalbashari, N. Freidoonimehr, F. Nazar, and M. M. Rashidi, "Analytical modelling of entropy generation for casson nano-fluid flow induced by a stretching surface," Advanced Powder Technology, vol. 26, no. 2, pp. 542-552, 2015.

[11] M. Khan and W. A. Khan, "MHD boundary layer flow of a power-law nano-fluid with new mass flux condition," AIP Advances, vol. 6, no. 2, Article ID 025211, 2016.

[12] S. Nadeem and S. T. Hussain, "Flow and heat transfer analysis of williamson nanofluid," Applied Nanoscience, vol. 4, no. 8, pp. 1005-1012, 2014.

[13] T. Hayat, Z. Hussain, A. Alsaedi, and B. Ahmad, "Heterogeneous-homogeneous reactions and melting heat transfer effects in flow with carbon nanotubes," Journal of Molecular Liquids, vol. 220, pp. 200-207, 2016.

[14] N. S. Akbar, "Biological analysis of nano Prandtl fluid model in diverging tube," Journal of Computational and Theoretical Nanoscience, vol. 12, no. 1, pp. 105-112, 2015.

[15] A. N. Sher, S. Nadeem, R. U. Haq, and Z. H. Khan, "Numerical solutions of magnetohydrodynamic boundary layer flow of tangent hyperbolic fluid towards a stretching sheet," Indian Journal of Physics, vol. 87, no. 11, pp. 1121-1124, 2013.

[16] A. J. Friedman, S. J. Dyke, and B. M. Phillips, "Over-driven control for large-scale MR dampers," Smart Materials and Structures, vol. 22, no. 4, Article ID 045001, pp. 4-10, 2013.

[17] S. Nadeem and S. Akram, "Peristaltic transport of a hyperbolic tangent fluid model in an asymmetric channel," Zeitschrift für Naturforschung A, vol. 64, no. 9-10, pp. 559-567, 2010.

[18] M. Naseer, M. Y. Malik, S. Nadeem, and A. Rehman, "The boundary layer flow of hyperbolic tangent fluid over a vertical exponentially stretching cylinder," Alexandria Engineering Journal, vol. 53, no. 3, pp. 747-750, 2014.

[19] S. Jyothi, M. V. Subba Reddy, and P. Gangavathi, "Hyperbolic tangent fluid flow through a porous medium in an inclined channel with peristalsis," International Journal of Advanced Scientific Research and Management, vol. 1, no. 4, pp. 113-121, 2016.

[20] H. Alfvén, "Existence of electromagnetic-hydrodynamic waves," Nature, vol. 150, pp. 405-406, 1942.

[21] N. S. Akbar, S. Nadeem, R. Haq, and Z. H. Khan, "Numerical solutions of Magnetohydrodynamic boundary layer flow of tangent hyperbolic fluid towards a stretching sheet," Indian Journal of Physics, vol. 87, pp. 1121-1124, 2013.

[22] X. Y. Tian, B. W. Li, Y. S. Wu, and J. K. Zhang, "Chebyshev collocation spectral method simulation for the 2D boundary layer flow and heat transfer in variable viscosity MHD fluid over a stretching plate," International Journal of Heat and Mass Transfer, vol. 89, pp. 29-37, 2018.

[23] M. Y. Malik, I. Khan, A. Hussain, and T. Salahuddin, "Mixed convection flow of MHD Eyring-Powell nanofluid over a stretching sheet: a numerical study," AIP Advances, vol. 5, no. 11, Article ID 117118, 2012.

[24] M. Y. Malik, M. Khan, T. Salahuddin, and I. Khan, "Variable viscosity and MHD flow in Casson fluid with Cattaneo -Christov heat flux model: using Keller box method," Engineering Science and Technology, an International Journal, vol. 19, pp. 1985-1992, 2016.

[25] S. Bilal, M. Y. Malik, M. Awais, K. Rehman, A. Hussain, and I. Khan, "Numerical investigation on $2 \mathrm{D}$ viscoelastic fluid due to exponentially stretching surface with magnetic effects: an application of non-Fourier flux theory," Neural Computing and Application, vol. 30, no. 2, pp. 2749-2758, 2018.

[26] C. S. Raju, M. J. Babu, and N. Sandeep, "Chemically reacting radiative MHD Jeffrey nanofluid flow over a cone in porous medium," International Journal of Engineering Research in Africa, vol. 19, pp. 75-90, 2015.

[27] G. V. P. N. Srikanth, B. S. Babu, G. Srinivas, and B.R.K. Reddy, "Heat and mass transfer of a MHD nanofluid with chemical reaction effects," International Journal of Mechanical and Production Engineering, vol. 2, pp. 2085-2092, 2014.

[28] M. R. Zangooee, K. Hosseinzadeh, and D. D. Ganji, "Hydrothermal analysis of MHD nanofluid $\left(\mathrm{TiO}_{2}-\mathrm{GO}\right)$ flow between two radiative stretchable rotating disks using AGM," Case Studies in Thermal Engineering, vol. 14, Article ID 100460, 2019.

[29] K. Hosseinzadeh, A. J. Amiri, S. S. Ardahaie, and D. D. Ganji, "Effect of variable Lorentz forces on nanofluid flow in movable parallel plates utilizing analytical method," Case Studies in Thermal Engineering, vol. 10, pp. 595-610, 2017.

[30] S. S. Ghadikolaei, K. Hosseinzadeh, and D. D. Ganji, "Investigation on ethylene glycol-water mixture fluid suspend by hybrid nanoparticles $\left(\mathrm{TiO}_{2}-\mathrm{CuO}\right)$ over rotating cone with considering nanoparticles shape factor," Journal of Molecular Liquids, vol. 272, pp. 226-236, 2018.

[31] M. Khan, M. Y. Malik, T. Salahuddin, K. U. Rehman, M. Naseer, and I. khan, "MHD flow of williamson nanofluid over a cone and plate with chemically reactive species," Journal of Molecular Liquids, vol. 231, pp. 580-588, 2017. 